\title{
Le Système intégré de surveillance des maladies chroniques du Québec (SISMACQ), une approche novatrice
}

\author{
C. Blais, Ph. D. (1, 2); S. Jean, Ph. D. (1, 3, 4); C. Sirois, Ph. D. (1, 5); L. Rochette, M. Sc. (1); C. Plante, M. Sc. (1); \\ I. Larocque, M. Sc. (1); M. Doucet, Ph. D. (1, 3); G. Ruel, Ph. D. (1,6); M. Simard, M. Sc. (1); P. Gamache, B. Sc. (1); \\ D. Hamel, M. Sc. (1); D. St-Laurent, M. Sc. (1); V. Émond, M. Sc. (1)
}

Cet article a fait l'objet d'une évaluation par les pairs.

\section{Résumé}

Introduction : Avec l'accroissement du fardeau des maladies chroniques, la surveillance est fondamentale pour améliorer la prévention et la prise en charge de ces dernières. L'Institut national de santé publique du Québec a donc développé un système novateur de surveillance des maladies chroniques, le Système intégré de surveillance des maladies chroniques du Québec (SISMACQ), dont les principales caractéristiques, les forces et les limites sont présentées ici.

Méthodologie : Le SISMACQ est le résultat du jumelage de cinq fichiers médicoadministratifs. Mises à jour annuellement, ses données couvrent actuellement la période du $1^{\text {er }}$ janvier 1996 au 31 mars 2012. Trois étapes en caractérisent le modèle opérationnel : 1) l'extraction et le jumelage des données médico-administratives grâce à divers critères de sélection; 2) les analyses (principalement la validation des définitions) et la production des mesures de surveillance et 3) l'interprétation, le dépôt et la diffusion de l'information. Le SISMACQ permet actuellement l'étude des maladies chroniques suivantes : diabète, maladies cardiovasculaires, maladies respiratoires, ostéoporose, maladies ostéoarticulaires, troubles mentaux et Alzheimer et maladies apparentées. Il permet également l'analyse de la multimorbidité et de la polypharmacie.

Résultats : Pour 2011-2012, le SISMACQ contenait des données sur 7995963 Québécois, et leur moyenne d'âge était de 40,8 ans. Parmi eux, 95,3\% répondaient à au moins un critère de sélection permettant l'application de définitions de cas pour la surveillance des maladies chroniques. Cette proportion variait avec l'âge : de 90,1 \% chez les Québécois de 19 ans et moins à 99,3 \% chez ceux de 65 ans et plus.

Conclusion : Le SISMACQ permet la production de données, à l'échelle de la population, sur le fardeau de plusieurs maladies chroniques, sur l'utilisation des services de santé et sur la consommation de médicaments. Il rend possible l'étude intégrée de la combinaison de plusieurs maladies, une approche jusqu'à présent rarement mise en œuvre dans un contexte de surveillance populationnelle. Le SISMACQ répond aux attributs essentiels d'un système de surveillance et aide à la diffusion de l'information auprès des décideurs en vue d'actions en santé publique.

Mots-clés : surveillance, maladies chroniques, fichiers médico-administratifs, modèle de surveillance, santé publique
Diffuser cet article sur Twitter

\section{Introduction}

D’après l'Organisation mondiale de la santé $(\mathrm{OMS})^{1}$ et les Nations unies ${ }^{2}$, le fardeau des maladies chroniques s'accroît et va continuer à s'accroître. Diverses stratégies de prévention et de contrôle sont nécessaires pour lutter contre cet état de fait, et le développement de la surveillance est le fondement de ces stratégies ${ }^{3}$. En effet, les données issues de la surveillance permettent d'identifier des sousgroupes d'individus atteints de maladies chroniques (ou à haut risque de les développer) et de cerner leurs besoins. Elles servent de guide lors de la mise en place des programmes de prévention, facilitent la planification des services de santé et orientent le choix des priorités en santé publique.

Le vieillissement de la population que connaît le Québec entraîne une charge importante sur le système de santé en regard des maladies chroniques. Celles-ci doivent ainsi faire l'objet d'une surveillance accrue $^{4}$, particulièrement les plus fréquentes, invalidantes, ou celles associées à une mortalité prématurée, telles que le diabète, les maladies cardiovasculaires (MCV), les maladies respiratoires, les maladies ostéoarticulaires, l'ostéoporose, les troubles mentaux ainsi que l'Alzheimer et les maladies apparentées. C'est dans ce contexte que le ministère de la Santé et des Services sociaux (MSSS) du Québec a mandaté l'Institut national de

Rattachement des auteurs :

1. Institut national de santé publique du Québec, Québec (Québec), Canada

2. Faculté de pharmacie, Université Laval, Québec (Québec), Canada

3. Faculté de médecine, Université Laval, Québec (Québec), Canada

4. Département de médecine, Université de Sherbrooke, Sherbrooke (Québec), Canada

5. Département de sciences infirmières, Université du Québec à Rimouski, Lévis (Québec), Canada

6. Population research outcome studies (PROS), University of Adelaide, Adelaide (South Australia), Australie

Correspondance : Valérie Émond, Institut national de santé publique du Québec, 945, avenue Wolfe, Québec (Québec) G1V 5B3; tél. : 418-650-5115, poste 5720; téléc. : 418-643-5099; courriel : valerie.emond@inspq.qc.ca 
santé publique du Québec (INSPQ) pour développer et mettre en œuvre la surveillance des maladies chroniques au Québec à partir de données médico-administratives.

Dans un système de santé universel comme celui du Québec, les données sur la santé colligées à des fins administratives constituent en effet une source précieuse pour la surveillance des maladies chroniques ${ }^{5}$. Ces données permettent l'obtention de mesures fiables et valides $^{6,7}$, de façon continue et systématique. De plus, le jumelage de plusieurs bases de données permet l'étude des issues de santé et de l'utilisation des ressources. Leur exploitation s'avère pratique, relativement simple, accessible et peu coûteuse. Généralisables à l'échelle de la population, elles permettent de minimiser les biais associés aux études réalisées sur la base d'échantillons (en particulier les biais de sélection, de rappel et de non-réponse) ${ }^{8,9}$. Cependant, comme ces fichiers de données sont conçus pour répondre à des besoins d'ordre administratif, leur utilisation à des fins de surveillance épidémiologique nécessite de respecter des standards de qualité rigoureux.

C'est ainsi que l'INSPQ a créé le Système intégré de surveillance des maladies chroniques du Québec (SISMACQ), dont nous dressons ici le portrait. Nous présentons son contenu et nous en décrivons les forces, les limites et les possibilités. Le choix d'une approche intégrée lors de la création du système s'est avéré essentiel pour répondre non seulement aux objectifs traditionnels de surveillance des maladies individuelles, mais également à ceux liés au cumul des maladies. Initialement développée pour la surveillance du diabète, cette méthode a permis également au Québec de participer, avec les autres provinces et territoires, au Système national de surveillance du diabète (SNSD), qui visait à combler des lacunes en matière d'information sur la prévalence et les effets du diabète au Canada ${ }^{10-12}$. Cette méthodologie a depuis été mise à profit pour la surveillance d'autres maladies chroniques, notamment au sein du Système canadien de surveillance des maladies chroniques (SCSMC).

\section{Méthodologie}

\section{Sources des données}

Les données du SISMACQ sont extraites de cinq fichiers médico-administratifs jumelés et mis à jour annuellement (figure 1, partie du haut) : le fichier d'inscription des personnes assurées (FIPA), le fichier des hospitalisations MED-ÉCHO (Maintenance et exploitation des données pour l'étude de la clientèle hospitalière), le fichier des décès du registre des événements démographiques, le fichier des services médicaux rémunérés à l'acte et le fichier des services pharmaceutiques (pour les personnes de 65 ans et plus). Parmi l'ensemble des variables présentes dans ces fichiers, seules celles nécessaires à la surveillance des maladies chroniques sont intégrées au
FIGURE 1

Modèle opérationnel du SISMACQ

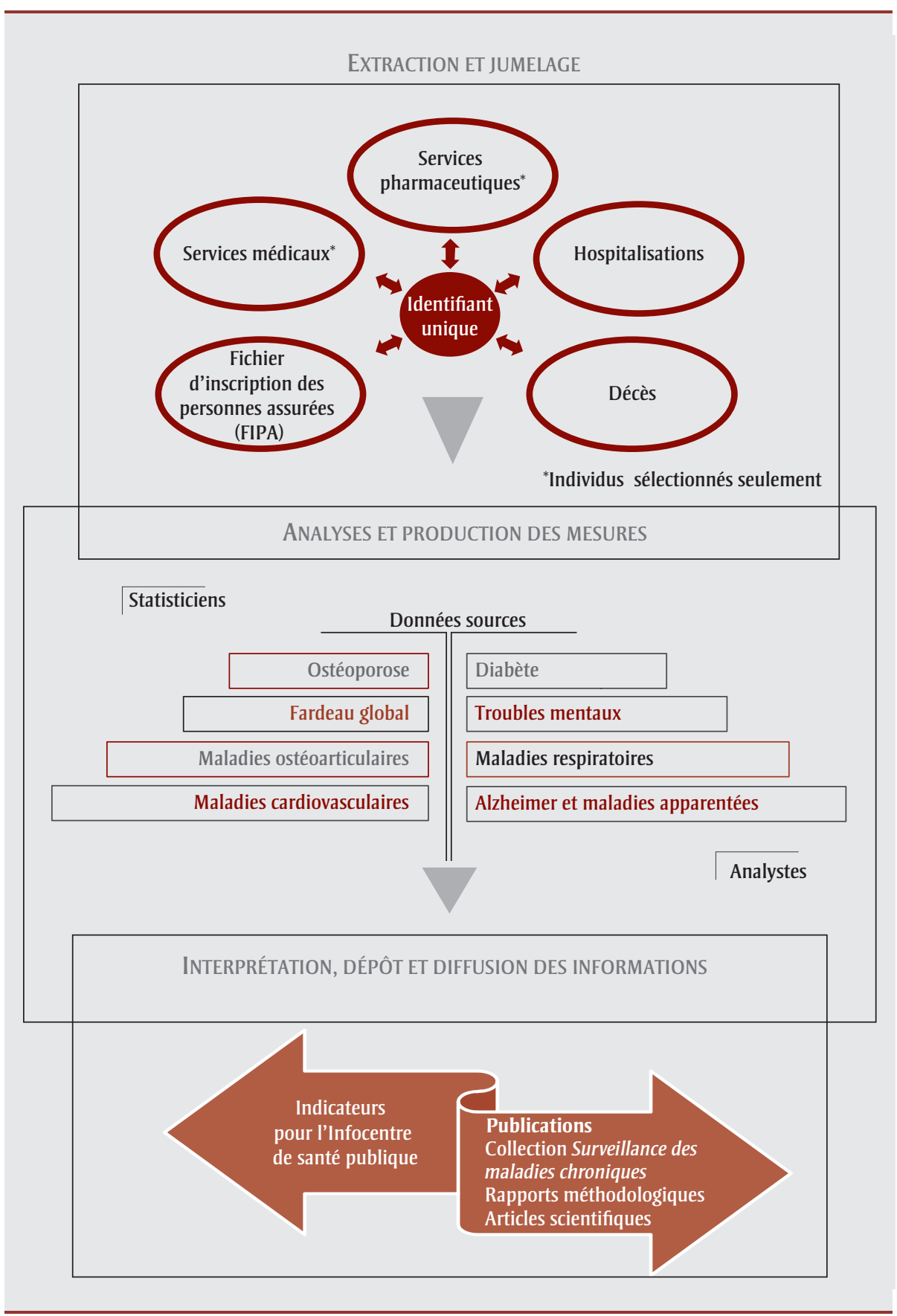

Abréviation : SISMACQ, Système intégré de surveillance des maladies chroniques du Québec. 
SISMACQ. Le numéro d'assurance maladie (NAM) est utilisé comme clé de jumelage des fichiers. À l'automne 2013, les données disponibles couvraient la période du $1^{\mathrm{er}}$ janvier 1996 au 31 mars 2012, hormis le fichier des décès (jusqu'au 31 décembre 2009).

- Le FIPA, administré par la Régie de l'assurance maladie du Québec $(\mathrm{RAMQ})^{13}$, contient des données individuelles démographiques et géographiques sur les personnes ayant un NAM valide et en vigueur, ainsi que des renseignements sur leur éligibilité et leur admissibilité aux régimes publics d'assurance maladie et d'assurance médicaments.

- Le fichier des hospitalisations (MEDÉCHO) regroupe l'information relative aux séjours en centres hospitaliers québécois dispensant des soins généraux ou spécialisés. Les données concernent les soins de courte durée et les chirurgies d'un jour et elles portent sur 1) le séjour hospitalier lui-même (lieu, durée, provenance et destination de l'individu), 2) les diagnostics (diagnostic d'admission, diagnostic principal, diagnostics secondaires et diagnostic de décès), 3) les services (décrits en fonction du lieu [chambre], de la spécialité du médecin ou du diagnostic), 4) les soins intensifs et 5) les interventions (thérapeutiques, diagnostiques, chirurgicales et obstétricales). Les diagnostics sont codés selon la neuvième Classification internationale des maladies (CIM-9) jusqu'au 31 mars 2006, et selon la CIM-10-CA depuis. Cette dernière répertorie 25 diagnostics secondaires, contre 15 pour la CIM-9. Les interventions sont codées selon la Classification canadienne des actes diagnostiques, thérapeutiques et chirurgicaux (CCADTC) en association avec la CIM-9 et selon la Classification canadienne des interventions en santé (CCI) avec la CIM-10-CA. Le nombre d'interventions est de 10 avec la CCADTC et de 20 avec la CCI. Ce sont des archivistes médicaux qui codent les données sommaires fournies par les médecins.

- Le fichier des décès enregistre tous les décès de Québécois, y compris ceux survenus à l'extérieur de la province.
L'information provient du médecin qui constate le décès ou du coroner et elle inclut la date du décès, sa cause principale et, depuis le $1^{\text {er }}$ janvier 2000, jusqu'à 10 causes contributives. Avant cette date, une seule cause additionnelle pouvait être inscrite en lien avec les décès attribuables à une cause externe. Les causes de décès sont codifiées conformément à la CIM, le $1^{\mathrm{er}}$ janvier 2000 étant la date de transition entre la CIM-9 et la CIM-10.

- Le fichier des services médicaux rémunérés à l'acte rassemble les données liées à la facturation à l'acte des professionnels, c'est-à-dire les demandes de paiement transmises à la RAMQ par les professionnels de la santé. Chaque enregistrement comprend le code de l'acte médical rendu et, optionnellement, dans $91 \%$ des réclamations de 1996-1997 à 2011-2012, un code de diagnostic associé codifié selon la CIM-9. Sont également présentes des données relatives aux professionnels de la santé eux-mêmes (professionnels traitant ou référant) et aux lieux des services.

- Le fichier des services pharmaceutiques centralise quant à lui les demandes de remboursement de médicaments dans le cadre du régime public d'assurance médicaments. Les données relevant des régimes privés d'assurance (auxquels souscrit une part importante de la population québécoise) ne s'y retrouvent donc pas. Les personnes de 65 ans et plus y sont présentes à plus de $90 \%$ (sauf celles couvertes par des régimes privés ou vivant en institution), en raison de l'admissibilité automatique au régime public à partir de cet âge. Sont inscrits les médicaments prescrits (code du médicament, dose, nombre de renouvellements, durée du traitement, etc.) et les informations sur les professionnels de la santé (classe du prescripteur, spécialité).

Étant donné que les fichiers contiennent peu d'information à caractère socioéconomique, le SISMACQ intègre l'indice de défavorisation matérielle et sociale développé à l'INSPQ, un substitut écologique du statut socioéconomique ${ }^{14}$. Créé à partir de six indicateurs du recensement canadien à l'échelle de l'aire de diffusion (AD), la plus petite unité géographique pour laquelle des données censitaires sont produites, l'indice est assigné à chaque individu grâce à un fichier de correspondance entre les $\mathrm{AD}$ et les codes postaux à six positions du FIPA. Les six indicateurs retenus sont : 1) la proportion de personnes n'ayant pas obtenu un diplôme d'études secondaires, 2) le rapport emploipopulation, 3) le revenu personnel moyen, 4) la proportion de personnes veuves, séparées ou divorcées, 5) la proportion de personnes vivant seules et 6) la proportion de familles monoparentales. Les trois premiers indicateurs renseignent sur la dimension matérielle de cet indice, tandis que les trois derniers renseignent sur sa dimension sociale ${ }^{14}$.

\section{Modèle opérationnel}

Le modèle opérationnel du SISMACQ repose sur trois étapes, en lien avec les trois composantes des systèmes de surveillance : l'extraction et le jumelage des données, l'analyse et la production des mesures ainsi que l'interprétation et la diffusion de l'information. Sa description ayant été publiée ailleurs ${ }^{15}$, nous en offrons une présentation très synthétique ici.

\section{Extraction et jumelage des données médico- administratives}

L'INSPQ reçoit, pour les variables sélectionnées, des identifiants uniques anonymes avec une information individuelle complète pour toute la population du Québec pour trois des fichiers (FIPA, fichier des hospitalisations et fichier des décès) et, en raison de restrictions concernant l'accès à l'information, une information individuelle pour seulement les personnes à risque d'au moins l'une des maladies chroniques étudiées pour les deux autres fichiers. Ces personnes sont identifiées à partir de critères de sélection basés sur des codes de diagnostic, des codes d'actes, des codes d'interventions hospitalières ou des codes pharmaceutiques. Même si nous obtenons l'intégralité du fichier des décès et des hospitalisations, nous appliquons également des critères de sélection des individus à risque lors de l'extraction des données, ce qui permet d'obtenir un historique les concernant dans les autres fichiers. Les codes de diagnostic ou de décès utilisés pour la sélection sont présentés dans le tableau 1. Les listes de codes 


\section{TABLEAU 1}

Liste des codes diagnostiques ou de décès utilisés pour sélectionner les individus potentiellement atteints de maladies chroniques dans le SISMACQ

\begin{tabular}{lll} 
Maladie & \multicolumn{1}{c}{ CIM-9 } & \multicolumn{1}{c}{ CIM-10-CA/CIM-10 } \\
\hline Diabète & $250 ;$ 648.0; 648.8; 790.2 & E10-E14, O24, O99.8, R73 \\
Maladies cardiovasculaires & $272,278,305.0,305.1,357.5,362,362.11,362.3,390-459$, & E66, E78, F10, F17, G45, G62.1, H34, I00-199, J81, M30.3, \\
et facteurs de risque associés & $514,518.4,584.5,584.9,585,586.9,745-747,785-786$, & N17, N18-N19, Q20-Q28, R00-R09, R34, R54, R57, \\
& $788.5,797-799,989.84, ~ V 158.2, ~ V 451$ & R96-R98, T65.2, Z50.2, Z71.4, Z72.0, Z99.2 \\
Maladies respiratoires & $490-493,496,460-462,464-466,480-486$ & J20, J45-J46, J40-J44, J00-02, J04-06, J12-18, J21-22 \\
Ostéoporose & $733,805-814,818-825,827-829,905,731$ & M80-M81, S12, S22, S32, S42, S52, S62, S72, S82, S92, T02, \\
& & T08, T10, T12, T14, T911-T912, T921-T922, T931-T932, \\
Maladies ostéoarticulaires & $710-729,274,446,696$ & T940, M88 \\
Troubles mentaux, Alzheimer & $046,290-319,331,332,797$ & M00-M99 \\
et maladies apparentées & & F00-F99, A81, B24, G10, G20, G30, G31, G35 \\
\hline
\end{tabular}

Abréviations : CIM-9, Classification internationale des maladies, neuvième révision; CIM-10, Classification internationale des maladies, dixième révision; CIM-10-CA, Classification internationale des maladies, dixième révision, adaptation canadienne; SISMACQ, Système intégré de surveillance des maladies chroniques du Québec.

Remarque : Le fardeau global inclut toutes ces maladies.

de médicaments, d'actes et d'interventions hospitalières sont disponibles sur demande.

À l'INSPQ, certains critères sont appliqués au FIPA pour créer le registre des personnes assurées du SISMACQ. Les données d'un individu inscrit au FIPA pour l'année à l'étude sont conservées si les critères d'éligibilité et d'admissibilité de cet individu sont respectés pour au moins une journée au cours de l'année en question (c'est-à-dire que son NAM est en vigueur). Les périodes d'inéligibilité correspondent au décès, à l'émigration, à la période de transition avant l'immigration et aux départs de la province pour 6 mois ou plus. Sont exclues également les périodes d'inadmissibilité au cours desquelles une personne n'a pas de carte d'assurance maladie en vigueur. Toutefois, afin d'obtenir un dénombrement similaire aux données démographiques officielles, le critère d'admissibilité n'est pas appliqué aux femmes de 18 à 25 ans et aux hommes de 18 à 29 ans, car une proportion assez élevée de membres de ces groupes ne renouvelle pas leur carte. Soulignons que la majorité de la population québécoise est inscrite au FIPA. Par conséquent, le registre du FIPA peut servir d'outil de dénombrement de la population, car ses données sont similaires aux données démographiques officielles publiées par l'Institut de la statistique du Québec (ISQ) ${ }^{16}$.

Le processus de création du SISMACQ et l'accès aux données respectent tous deux des critères élevés de sécurité et de protection des renseignements personnels. Aussi bien les organismes gouvernementaux possédant légalement les fichiers (RAMQ et MSSS) que le comité d'éthique de santé publique et la Commission d'accès à l'information du Québec ont évalué et approuvé ce processus de création. Les données sont entreposées dans un serveur sécurisé à l'INSPQ. L'accès aux données est journalisé et il est limité au personnel autorisé de l'Unité de surveillance des maladies chroniques et des traumatismes, selon différents niveaux d'accès.

\section{Analyses et production des mesures}

L'identification des personnes atteintes d'une maladie chronique se fait en utilisant des définitions de cas spécifiques à chaque maladie. Les études de validation sont essentielles afin de sélectionner des définitions qui minimisent les erreurs de classification. Le tableau 2 présente quelques exemples de définitions de cas appliquées aux données du SISMACQ, ainsi que les références des études de validation $^{17-21}$.

Grâce à sa large couverture de la population du Québec, le SISMACQ permet de décrire l'ampleur des maladies chroniques aux échelles provinciale et régionale, en fonction de divers déterminants (âge, sexe, défavorisation), d'en analyser les évolutions et de réaliser des projections. L’approche intégrée favorise le développe- ment de nouveaux indicateurs portant sur les issues d'une maladie (excès de mortalité, taux de complications), la comparaison de l'utilisation des services hospitaliers entre les personnes atteintes d'une maladie chronique et celles n'en étant pas atteintes ainsi que les analyses sur le continuum de la maladie et les trajectoires de soins. Le SISMACQ offre également, outre la production de mesures de surveillance conventionnelles, la possibilité de produire des mesures novatrices, notamment sur la multimorbidité et la polypharmacie.

\section{Interprétation, dépôt et diffusion des informations}

L'interprétation des données et le transfert des connaissances font partie intégrante de la surveillance ${ }^{5,22}$. Parmi les modes de diffusion associés au SISMACQ, mentionnons la transmission de données agrégées aux différents acteurs de la santé publique (notamment les régions sociosanitaires) à partir du site Internet sécurisé de l'Infocentre de santé publique du Québec, l'envoi de données agrégées sommaires à l'Agence de la santé publique du Canada dans le cadre du SCSMC et la publication de différents types de documents (par exemple, des séries thématiques, dont la collection Surveillance des maladies chroniques, des rapports méthodologiques et des articles scientifiques).

\section{Résultats}

Le premier dépôt de données du SISMACQ à l'INSPQ, effectué en 2010, portait sur la 
TABLEAU 2

Exemples de définitions de cas utilisées dans le SISMACQ

\begin{tabular}{|c|c|c|c|c|c|c|}
\hline \multirow[t]{2}{*}{ Maladie } & \multirow[t]{2}{*}{ Âge (ans) } & \multirow[t]{2}{*}{ Définition de cas } & \multicolumn{2}{|c|}{ Code diagnostique } & \multirow[t]{2}{*}{ Intervention } & \multirow{2}{*}{$\begin{array}{l}\text { Études de } \\
\text { validation }\end{array}$} \\
\hline & & & CIM-9 & CIM-10-CA & & \\
\hline $\begin{array}{l}\text { Cardiopathies } \\
\text { ischémiques }\end{array}$ & 20 et plus & $\begin{array}{l}\text { Deux diagnostics de cardiopathies ischémiques } \\
\text { inscrits au fichier des services médicaux } \\
\text { rémunérés à l'acte en } 1 \text { an ( } 365 \text { jours) } \\
\text { OU } \\
\text { un diagnostic (principal ou secondaire) } \\
\text { OU } \\
\text { un code d'intervention des cardiopathies } \\
\text { ischémiques enregistré au fichier MED-ÉCHO }\end{array}$ & 410 à 414 & 120 à 125 & $\begin{array}{l}\text { CCADTC }^{\mathrm{a}} \\
48.02,48.03, \\
48.11-48.19 \\
\text { CCI }^{\mathrm{b}} \\
\text { 1.IJ.50, } \\
\text { 1.IJ.57.GQ, } \\
\text { 1.IJ.54, 1.IJ.76 }\end{array}$ & $\begin{array}{l}\text { Tu et collab., } \\
2010^{19}\end{array}$ \\
\hline $\begin{array}{l}\text { Diabète, } \\
\text { hypertension }\end{array}$ & $\begin{array}{l}1 \text { et plus } \\
\text { (diabète), } \\
20 \text { et plus } \\
\text { (hypertension) }\end{array}$ & $\begin{array}{l}\text { Deux diagnostics de diabète (ou d'hypertension) } \\
\text { inscrits au fichier des services médicaux } \\
\text { rémunérés à l'acte en } 2 \text { ans ( } 730 \text { jours) } \\
\text { OU } \\
\text { un diagnostic (principal ou secondaire) de } \\
\text { diabète (ou d'hypertension) enregistré au } \\
\text { fichier MED-ÉCHO } \\
\text { Exclusion des cas de diabète } \\
\text { (ou d'hypertension) gestationnel }\end{array}$ & $\begin{array}{l}250 \\
\text { (diabète) } \\
401-405 \\
\text { (hypertension) }\end{array}$ & $\begin{array}{l}\text { E10 à E14 } \\
\text { (diabète) } \\
\text { I10 à I15 } \\
\text { (hypertension) }\end{array}$ & & $\begin{array}{l}\text { Ouhoummane } \\
\text { et collab., } \\
2010^{44} \\
\text { Hux et collab., } \\
2002^{17} \\
\text { Quan et collab., } \\
2009^{20} \\
\text { Tu et collab., } \\
2007^{21}\end{array}$ \\
\hline $\begin{array}{l}\text { Fractures } \\
\text { ostéoporotiques }\end{array}$ & 50 et plus & $\begin{array}{l}\text { Un acte médical inscrit au fichier des services } \\
\text { médicaux rémunérés à l'acte correspondant } \\
\text { à une réduction ouverte, une réduction } \\
\text { fermée ou une immobilisation de fracture } \\
\text { OU } \\
\text { un acte médical de visite principale ou d'une } \\
\text { consultation avec orthopédiste avec un } \\
\text { diagnostic de fracture + au moins un } \\
\text { autre acte médical de consultation avec un } \\
\text { diagnostic de fracture au même site en } 4 \text { mois }\end{array}$ & & Voir référence & & $\begin{array}{l}\text { Jean et collab., } \\
2012^{18}\end{array}$ \\
\hline
\end{tabular}

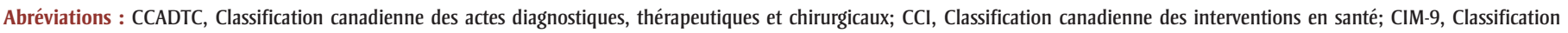

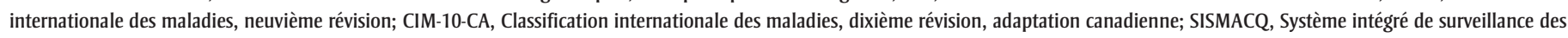
maladies chroniques du Québec.

${ }^{a}$ La CCADTC est associée à la CIM-9 dans le fichier des hospitalisations avant le $1^{\text {er }}$ avril 2006.

b La CCI est associée à la CIM-10-CA dans le fichier des hospitalisations.

période du $1^{\text {er }}$ janvier 1996 au 31 mars 2009. Depuis, une mise à jour annuelle est effectuée chaque été. Ainsi, à l'automne 2013, la couverture du SISMACQ s'étendait jusqu'au 31 mars 2012.

Le tableau 3 présente diverses caractéristiques de la population inscrite au registre des personnes assurées du SISMACQ. Pour l'année financière 2011-2012, 7995963 Québécois ont été éligibles et admissibles à la RAMQ (moyenne d'âge de 40,8 ans), ce qui représente 99,1\% de la population selon les données officielles ${ }^{16}$. La part de la population âgée de 65 ans et plus était de $16,0 \%$. Cette proportion a augmenté entre 2001-2002 et 2011-2012. Plus de la moitié de ce sous-groupe était constitué de femmes $(56,2 \%)$ et $89,9 \%$ étaient inscrits à l'assurance médicaments. Près de la moitié des Québécois vivaient dans la région métropolitaine de recensement de Montréal (48,2 \%). Seule la population vivant en zone rurale tend à diminuer légèrement avec le temps. Entre $2,5 \%$ des individus en 2001-2002 et 1,3\% en 2011-2012 ne sont pas associés à une zone géographique, en raison de l'absence du code postal ou d'un code postal erroné. Bien que la proportion de personnes inscrites à l'assurance médicaments diminue légèrement entre 2001-2002 et 2011-2012, le nombre d'inscrits augmente.

Les individus potentiellement atteints d'au moins l'une des maladies chroniques à l'étude ont été sélectionnés dans les différents fichiers médico-administratifs à partir de plusieurs critères. Le tableau 4 présente le nombre et la proportion d'individus répondant à au moins un critère de sélection, en fonction de leur âge et de l'année. Une grande proportion de la population, particulièrement au sein des tranches d'âge les plus élevées, est incluse dans le SISMACQ, étant donné la prévalence élevée de plusieurs maladies chroniques et le choix des critères de sélection. Pour 2001-2002, ce sont 97,9\% des inscrits au registre des personnes assurées qui répondent à au moins un critère de sélection et cette proportion atteint 99,7 \% pour les personnes de 65 ans ou plus. Pour 2011-2012, 95,3 \% des individus répondent à au moins un critère de sélection. Cette légère diminution par rapport à 2001-2002 pourrait être attribuable à une période de suivi plus courte, ce qui diminue la probabilité de satisfaire à au moins un critère de sélection, entre autres chez les jeunes (90,1\% chez les 19 ans et moins). Rappelons que les individus satisfaisant aux critères de sélection sont potentiellement à risque, mais que tous ne sont pas affectés par une maladie chronique. Par exemple, pour la 


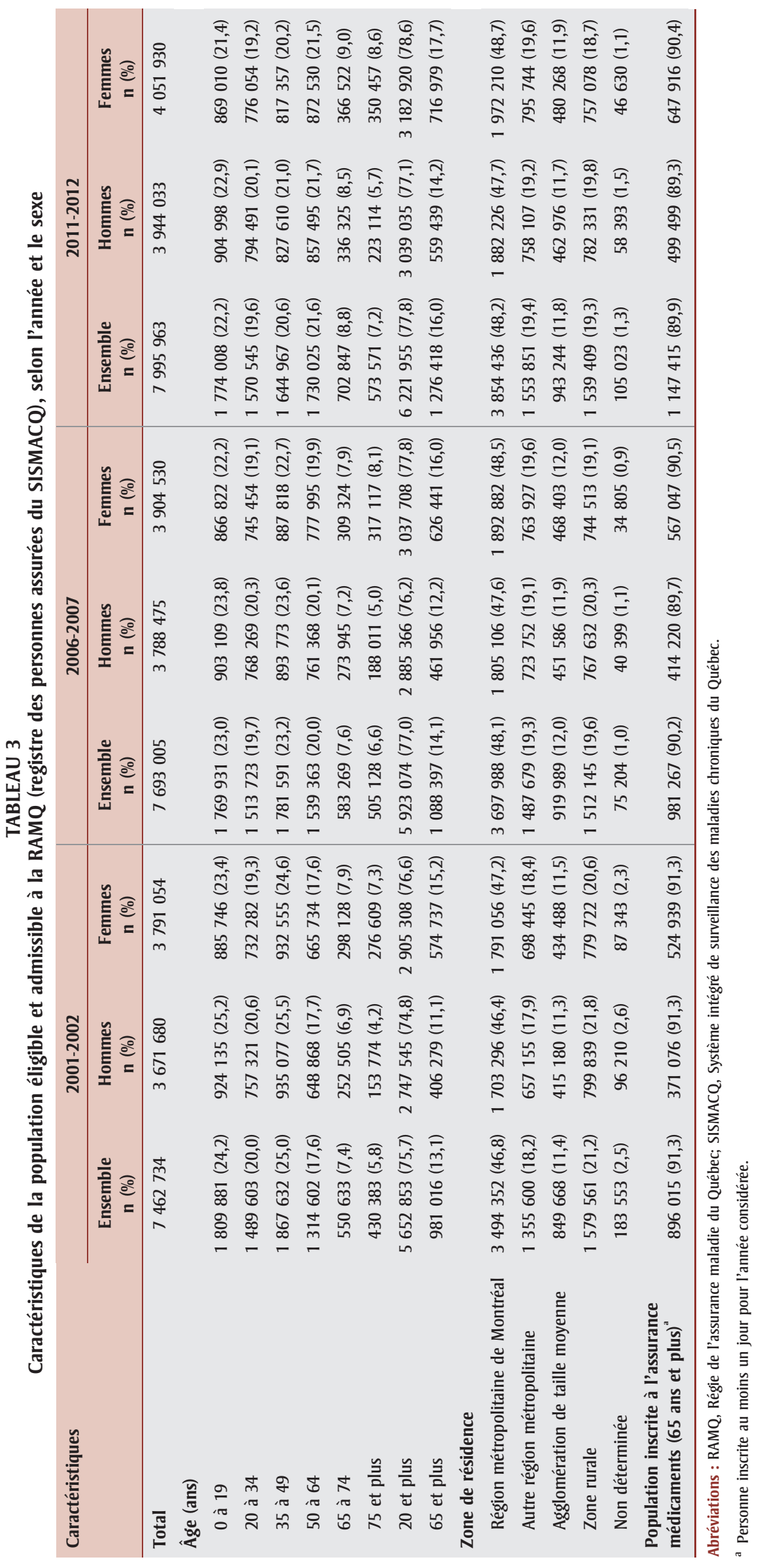

surveillance des MCV, les critères de sélection ont permis d'identifier 6164006 $(77,1 \%)$ individus en 2011-2012. Dans les faits, pour cette même année, 1483168 individus de 20 ans et plus $(23,8 \%$ prévalence brute) ont satisfait la définition de cas de l'hypertension artérielle présentée dans le tableau 2, permettant d'évaluer l'ampleur de cette problématique en termes d'incidence et de prévalence.

\section{Analyse}

La surveillance représente une étape fondamentale dans la mesure de l'évolution de l'état de santé de la population. Au Québec, la cueillette de données sur les événements de santé a évolué et touche aujourd'hui principalement trois aspects : la collecte elle-même, l'analyse et l'interprétation de ces données et la diffusion en temps opportun de l'information auprès des décideurs en vue du contrôle et de la prévention des maladies. Les sources de données les plus pertinentes que le Québec possède pour effectuer une surveillance des maladies chroniques sont les fichiers médico-administratifs : ils sont alimentés de façon continue et systématique, ils peuvent être jumelés et ils nécessitent peu d'efforts ou de coûts supplémentaires.

Le SISMACQ se révèle être ainsi une source inestimable d'information sur le fardeau des maladies chroniques au Québec, puisqu'il couvre l'ensemble des services de santé utilisés par la population, de la consultation médicale au décès en passant par l'hospitalisation et la consommation de médicaments. La mise en place de ce système permet une surveillance des maladies chroniques actualisée, qui tient compte de leur cooccurrence et de l'organisation des soins et des services, ce qui est très important dans un contexte de vieillissement de la population. En effet, les données du SISMACQ répondent à des besoins grandissants en information sur les populations vulnérables face à la maladie chronique ${ }^{23-24}$. Avec des données disponibles depuis 1996 et des mises à jour annuelles, ce système facilite les analyses d'utilisation de services $^{25}$, de tendance et de projections à partir de cohortes populationnelles ${ }^{26,27}$. L'information obtenue à partir du 
TABLEAU 4

Nombre et proportion d'individus répondant à au moins un critère de sélection ${ }^{\mathrm{a}}$ du SISMACQ, selon l'année et l'âge

\begin{tabular}{|c|c|c|c|c|c|c|}
\hline \multirow[t]{2}{*}{ Caractéristiques } & \multicolumn{2}{|c|}{ 2001-2002 } & \multicolumn{2}{|c|}{ 2006-2007 } & \multicolumn{2}{|c|}{ 2011-2012 } \\
\hline & $\begin{array}{c}\text { Total (registre des } \\
\text { personnes assurées) } \\
\mathbf{n}\end{array}$ & $\begin{array}{c}\text { Au moins un critère } \\
\text { de sélection } \\
\text { n (\%) }\end{array}$ & $\begin{array}{c}\text { Total (registre des } \\
\text { personnes assurées) } \\
\text { n }\end{array}$ & $\begin{array}{c}\text { Au moins un critère } \\
\text { de sélection } \\
\text { n (\%) }\end{array}$ & $\begin{array}{c}\text { Total (registre des } \\
\text { personnes assurées) } \\
\text { n }\end{array}$ & $\begin{array}{c}\text { Au moins un critère } \\
\text { de sélection } \\
\text { n (\%) }\end{array}$ \\
\hline Total & 7462734 & $7307855(97,9)$ & 7693005 & $7511892(97,6)$ & 7995963 & $7617930(95,3)$ \\
\hline \multicolumn{7}{|l|}{ Âge (ans) } \\
\hline 0 à 19 & 1809881 & $1764479(97,5)$ & 1769931 & $1710415(96,6)$ & 1774008 & $1599191(90,1)$ \\
\hline 35 à 49 & 1867632 & $1830421(98,0)$ & 1781591 & $1744230(97,9)$ & 1644967 & $1586085(96,4)$ \\
\hline 50 à 64 & 1314602 & $1299876(98,9)$ & 1539363 & $1519958(98,7)$ & 1730025 & $1700141(98,3)$ \\
\hline 65 et plus & 981016 & $978054(99,7)$ & 1088397 & $1084196(99,6)$ & 1276418 & $1267763(99,3)$ \\
\hline
\end{tabular}

Abréviation : SISMACQ, Système intégré de surveillance des maladies chroniques du Québec.

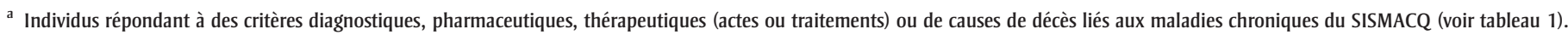

SISMACQ contribue à la compréhension du continuum allant de la prévention de la maladie à sa progression, ses traitements et les résultats (effets des programmes de santé publique, consommation de soins, adhésion aux traitements, etc.), ce qui aide à planifier les ressources et les interventions en santé publique.

\section{Attributs essentiels et forces du SISMACQ}

Le SISMACQ répond très bien aux cinq exigences de base des systèmes de surveillance en santé publique $e^{5,22,28}$ : 1) simplicité et flexibilité, 2) acceptabilité, 3) sensibilité et valeur prédictive positive, 4) représentativité et enfin 5) promptitude.

- Dans un contexte où les ressources humaines et matérielles sont limitées, l'utilisation secondaire de données recueillies à des fins administratives est relativement simple et peu coûteuse. Par l'ajout annuel de nouveaux codes de sélection et de cas potentiels, le SISMACQ demeure flexible. Cependant, cette flexibilité est limitée en raison des contraintes légales. De plus, le système ne permet pas de réagir rapidement à des changements dans l'organisation des soins et à des nouveautés comme l'ajout de maladies ou de sources de données (p. ex. : banque de données sur les groupes de médecine familiale).

- L'acceptabilité du SISMACQ est, pour sa part, excellente. Les organismes responsables des fichiers médicoadministratifs acceptent que les données soient utilisées pour la surveil- lance. Les décideurs utilisent déjà l'information issue de ce système et ils en réalisent la pertinence. De plus, les acteurs régionaux de surveillance, qui utilisent beaucoup les informations du SISMACQ, désirent s'impliquer activement dans les activités liées à ce système.

- Les mesures de validité que sont la sensibilité et la valeur prédictive positive varient d'une maladie à l'autre et dépendent des définitions de cas. Dans un contexte de surveillance à l'échelle d'une population, on recherche un équilibre entre ces deux mesures de validité pour chaque maladie et définition de cas.

- Le SISMACQ se distingue par ailleurs au niveau de la représentativité, puisqu'il jumelle plusieurs sources de données issues d'un système universel de santé. Sa couverture permet de généraliser l'information à l'ensemble de la population, de décrire les caractéristiques sociodémographiques, économiques et géographiques et de minimiser plusieurs biais de sélection. Cette capacité du SISMACQ à établir un lien entre différentes sources de données a également pour effet de rehausser la qualité et l'utilité de l'information qui en est issue.

- Quant à la promptitude, ce n'est pas un critère aussi critique pour la surveillance des maladies chroniques que pour d'autres systèmes de surveillance (p. ex. ceux portant sur les maladies infectieuses). Avec sa mise à jour annuelle, le SISMACQ peut produire des mesures d'incidence en temps opportun et ainsi contribuer à l'intervention dans des délais raisonnables.

En résumé, le SISMACQ répond adéquatement à l'ensemble des attributs exigibles d'un système de surveillance et, comme il est basé sur la consommation de services de santé d'un système universel, il répond au principe fondamental de la surveillance, c'est-à-dire la présence d'un système de santé fonctionnel ${ }^{5}$.

Parmi les autres forces du SISMACQ, mentionnons l'accès à des données sources, c'est-à-dire brutes, ce qui permet un contrôle de la qualité aux différentes étapes de traitement et d'analyse de ces données. En effet, bien que la RAMQ soit responsable d'appliquer elle-même certains des critères de sélection, d'extraction et de jumelage et qu'elle soit aussi responsable du cryptage du NAM, l'INSPQ reçoit tout de même les données sous forme d'enregistrements individuels. De plus, l'ajout de l'indice de défavorisation fait du SISMACQ une précieuse source d'information sur les impacts des inégalités sociales en santé ${ }^{23,24}$. Par ailleurs, le SISMACQ permet à l'INSPQ de produire des mesures agrégées de surveillance des maladies chroniques pour le Québec harmonisées avec celles des autres provinces et territoires canadiens participant également au SCSMC, une initiative coordonnée par l'Agence de la santé publique du Canada. Finalement, le fait que les données soient collectées à des fins administratives peut, notamment dans le cas du fichier des services pharmaceutiques, représenter un avantage pour la 
qualité. En effet, selon Tamblyn et collab. ${ }^{29}$, ce type de fichier fournit des données assez exactes et remarquablement complètes puisque les demandeurs peuvent se voir refuser leur paiement si des champs sont mal remplis dans leur réclamation.

\section{Comparaison du SISMACQ avec d'autres systèmes de surveillance}

Le SISMACQ tire avantage d'une comparaison avec les systèmes de surveillance déployés dans le monde. Aux États-Unis, l'Institute of Medicine a élaboré un cadre national de surveillance des maladies cardiovasculaires et pulmonaires chroniques et a critiqué sévèrement dans ce contexte l'absence d'un système de surveillance opérant sur une base nationale et susceptible d'outiller les décideurs en temps opportun $^{30}$. En effet, bien que les sources de données américaines comprennent, entre autres, des enquêtes de population (comme la National Health and Nutrition Examination Survey [NHANES]), des registres de maladies spécifiques, des cohortes populationnelles comme celle de Framingham ou de Rochester ${ }^{31}$ ou encore des données de réclamations déposées auprès de compagnies d'assurance, aucune de ces sources ne pouvait, jusqu'à tout récemment, être jumelée afin de fournir un portrait national et procurer de l'information sur l'incidence. Le National Center for Health Statistics jumelle présentement plusieurs enquêtes de population avec, entre autres, les certificats de décès, les données des services de Medicare and Medicaid et l'historique des données sur la sécurité sociale $^{32}$. L'Australie effectue quant à elle la majorité de sa surveillance à partir de données d'enquêtes, en particulier l'Australian Bureau of Statistics National Health Survey $(\mathrm{NHS})^{33}$. La stratégie de surveillance de la population du Royaume-Uni repose également sur l'intégration et l'exploitation de nouvelles sources de données comme les registres de maladies aigües et chroniques ainsi que sur le suivi des comportements liés à la santé (tabagisme, alimentation, exercice, etc. $)^{34}$. Étant donné sa flexibilité, on peut tout à fait envisager l'intégration d'information de ce type au SISMACQ. D'ailleurs, d'autres provinces, notamment l'Ontario ${ }^{35}$ et le Manitoba ${ }^{36}$, non seulement ont accès aux données jumelées des fichiers médicoadministratifs, mais intègrent également des données d'enquêtes dans leurs systèmes de surveillance.

\section{Limites du SISMACQ}

La principale limite du SISMACQ relève de la nature des sources de données utilisées. En effet, comme les fichiers médicoadministratifs ont été conçus par définition pour répondre à des besoins d'ordre administratif, une validation de la banque de données ${ }^{37}$ et des définitions de cas $^{17-19}$ est nécessaire dans le cas d'une utilisation en épidémiologie. Par ailleurs, même si les études de validation peuvent attester de la qualité de la définition de cas choisie, seules les personnes qui ont recours aux services de santé et qui sont diagnostiquées sont incluses dans les analyses, ce qui constitue une sous-estimation de la véritable ampleur des maladies. Par exemple, une personne pourrait ne pas savoir qu'elle a subi un infarctus du myocarde et ne jamais être allée consulter un médecin, de sorte que cet infarctus ne sera pas comptabilisé dans le SISMACQ ${ }^{38}$.

De plus, certaines données sont absentes des fichiers du SISMACQ, par exemple celles concernant les membres des Forces canadiennes, dont les services médicaux sont payés par le gouvernement fédéral ${ }^{39}$. Les actes des Québécois traités dans une autre province par des médecins non inscrits à la RAMQ ne se retrouvent pas non plus dans le SISMACQ actuellement. Des travaux sont cependant en cours afin d'intégrer des fichiers complémentaires sur les hospitalisations et les services reçus à l'extérieur de la province, ce qui permettrait de minimiser la sous-estimation des services reçus, particulièrement dans les régions limitrophes (par exemple l'Outaouais). Aucune information n'est disponible non plus sur l'hébergement pour soins de longue durée, et l'information pour les services pharmaceutiques est limitée aux personnes de 65 ans et plus.

Enfin, certains médecins québécois ne sont pas rémunérés à l'acte, ce qui entraîne une sous-estimation des services reçus et une diminution de la sensibilité du SISMACQ ${ }^{39}$. Les autres formes de paiement sont les rémunérations à salaire, à forfait (p. ex. prime de prise en charge pour les omnipraticiens), à honoraires forfaitaires (per diem ou à vacation) ou, depuis le $1^{\text {er }}$ septembre 1999, selon une rémunération mixte (à l'acte et à honoraires forfaitaires). Pour 2010-2011, l'ensemble des autres formes de rémunération représentait $24 \%$ de la rémunération des médecins, contre $16 \%$ pour 1999-2000. L'augmentation de la popularité de la rémunération mixte depuis son introduction en 1999 pour les spécialistes explique en grande partie ce changement : l'honoraire forfaitaire relié à ce type de rémunération représente maintenant 11,6\% de l'ensemble des paiements cliniques faits aux médecins, alors que, pour leur part, les salaires ne représentent que $1,9 \%^{40}$. Quoique les modes de rémunération autres que celui à l'acte soient proportionnellement en augmentation, le nombre de services médicaux à l'acte a augmenté de 8,8\% entre 2001 et 2007, l'année 2001 étant celle en présentant le plus faible nombre en 18 ans. Les effets des rémunérations alternatives se manifestent surtout dans les localités éloignées. Pour ces populations, des sources de données alternatives ou des enquêtes sont nécessaires pour améliorer la surveillance. Par exemple, dans les collectivités autochtones des Terres-Cries-de-la-Baie-James, où l'ensemble des omnipraticiens est rémunéré par des ententes alternatives, un projet pilote de jumelage avec le Système d'information sur le diabète cri (SIDC) a permis de constater que le SISMACQ n'identifiait que $60 \%$ des diabétiques de cette région, et avec un délai moyen de 2,3 années après la date d'incidence de la maladie. Ce jumelage ayant permis de limiter le manque d'information sur les autres modes de rémunération, il est envisagé de jumeler ces deux systèmes de manière récurrente. Par ailleurs, la présence du secteur privé dans le système de santé québécois demeure somme toute marginale et affecte principalement le fichier des services médicaux et le fichier des services pharmaceutiques pour les personnes de moins de 65 ans.

Enfin, la définition de chacune des maladies chroniques étudiées dans le SISMACQ a ses limites. L'étude de la multimorbidité s'en trouve ainsi complexifiée. Par ailleurs, les données médico-administratives ne permettent généralement pas de 
connaître la sévérité de la maladie ou de confirmer le diagnostic par des informations cliniques. Les fichiers médicoadministratifs renferment aussi très peu de données sur les facteurs de risque des maladies chroniques, et aucune information sur les résultats des examens de laboratoire ou sur les habitudes de vie ayant un lien avec les maladies chroniques (alimentation, activité physique, tabagisme, consommation d'alcool). Le fichier des hospitalisations se révèle cependant être une excellente source d'information pour certains facteurs de risque et certaines comorbidités, puisqu'il tient compte des diagnostics secondaires présents et de ceux ayant contribué au séjour hospitalier (25 possibilités depuis le $1^{\mathrm{er}}$ avril 2006).

\section{Conclusion}

Avec le vieillissement de la population et l'augmentation de l'espérance de vie, le fardeau des maladies chroniques va continuer de croître et de se complexifier. On doit donc actualiser la surveillance de ces dernières afin de connaître la population à risque et la population qui en est atteinte et afin de suivre l'évolution de ces maladies et les enjeux qui leur sont associés. Le SISMACQ se révèle être la façon la plus appropriée d'assurer cette surveillance au Québec. Il permet de maximiser l'utilisation des systèmes d'information disponibles, s'avère peu coûteux et fournit des données fiables à l'échelle de la population. Il contient des données individuelles, hospitalières, médicales et socioéconomiques et sur les causes de décès pour près de 8 millions de Québécois pour 2011-2012, de tous les âges et de toutes les régions, ce qui permet l'étude exhaustive du fardeau des principales maladies chroniques. Par ailleurs, le SISMACQ est conforme au modèle intégré de prévention et de gestion des maladies chroniques ${ }^{41}$. Dans cette perspective, la surveillance ne concerne pas seulement chaque maladie prise séparément, mais aussi le cumul de maladies et les complications qui lui sont associées.

Le SISMACQ remplit les fins d'un système de surveillance intégré, efficient et tourné vers l'avenir, que des bonifications futures pourront enrichir ${ }^{42}$. En effet, il faudra envisager d'intégrer de l'information sur les facteurs de risque et sur les habitudes de vie associés aux maladies chroniques. Est déjà prévu pour les années à venir le jumelage avec d'autres fichiers médicoadministratifs (notamment les fichiers des naissances et mortinaissances), avec des données d'enquête sur la santé et avec des systèmes d'information comme celui du diabète chez les Cris. Le modèle décrit dans ce document sera ainsi résolument évolutif et pourra s'adapter aux besoins exprimés. Notons pour terminer que, dans un souci de rigueur scientifique, le SISMACQ devra être régulièrement évalué afin de s'assurer que la surveillance effectuée avec ce système est efficiente et utile pour la prise de décision et l'action en santé publique.

\section{Remerciements}

Les auteurs remercient l'Agence de la santé publique du Canada et le ministère de la Santé et des Services sociaux du Québec pour leur contribution financière.

\section{Références}

1. Organisation mondiale de la santé (OMS). Prévention des maladies chroniques : un investissement vital. Genève : OMS; 2006.

2. Nations Unies. Déclaration politique de la Réunion de haut niveau de l'Assemblée générale sur la prévention et la maîtrise des maladies non transmissibles. New York (NY) : Nations Unies; 2012. PDF téléchargeable à partir de la page : http://www.un.org /fr/documents/view_doc.asp?symbol = A/66 $/ \mathrm{L} .1$

3. Santé Canada. La surveillance des maladies chroniques au Canada. Document de référence. Ottawa (Ont.) : Santé Canada; 2003.

4. Ministère de la Santé et des Services sociaux du Québec (MSSS), Institut national de santé publique du Québec (INSPQ). Plan ministériel de surveillance multithématique, Thème 1, Habitudes de vie, comportements et maladies chroniques, Volet 1 : Maladies chroniques. Québec (Qc) : MSSS et INSPQ; 2010.

5. Kone Pefoyo AJ, Rivard M, Laurier C. Importance de la surveillance en santé publique et utilité des données administratives. Rev Epidemiol Sante Publique. 2009;57(2):99-111.
6. Roos LL Jr., Roos NP, Cageorge SM, Nicol JP. How good are the data? Reliability of one health care data bank. Med Care. 1982;20(3):266-276.

7. Roos LL Jr., Gupta S, Soodeen RA, Jebamani L. Data quality in an information-rich environment: Canada as an example. Can J Aging. 2005;24 Suppl 1:153-170.

8. Benchimol EI, Manuel DG, To T, Griffiths AM, Rabeneck L, Guttmann A. Development and use of reporting guidelines for assessing the quality of validation studies of health administrative data. J Clin Epidemiol. 2011; 64(8):821-829.

9. Wilchesky M, Tamblyn RM, Huang A. Validation of diagnostic codes within medical services claims. J Clin Epidemiol. 2004; 57(2):131-141.

10. Émond V. Prévalence du diabète au Québec et dans ses régions : premières estimations d'après les fichiers administratifs. Québec (Qc) : Institut national de santé publique du Québec; 2002.

11. Patt A. Marshalling the Troops : Advocating for the Needs of People With Diabetes. Canadian Journal of Diabetes. 2005;29(3): 180.

12. Clottey C, Mo F, LeBrun B, Mickelson P, Niles J, Robbins G. La mise sur pied du Système national de surveillance du diabète (SNSD) au Canada. Maladies chroniques au Canada. 2001;22(2):75-77.

13. Régie de l'assurance maladie du Québec (RAMQ). Données et statistiques. Données sur demande [Internet]. Québec (Qc) : RAMQ; 2013 [consultation le 20 décembre 2013]. Consultable en ligne à la page : http://www.ramq.gouv.qc.ca/fr/donnees -statistiques/sur-demande/donnees-regie

14. Pampalon R, Hamel D, Gamache P, Raymond G. Un indice de défavorisation pour la planification de la santé au Canada. Maladies chroniques au Canada. 2009;29(4): 199-213.

15. Saint-Laurent D, Blais C, Jean S, Sirois C, Rochette L, Émond V. Le modèle québécois de surveillance des maladies chroniques basé sur l'utilisation des données médicoadministratives jumelées. Bulletin épidémiologique hebdomadaire. 2013; Numéro hors-série:4-8. 
16. Institut de la statistique du Québec (ISQ). Banque de données de statistiques officielles sur le Québec. Population et démographie [Internet]. Québec (Qc) : ISQ; 2013 [consultation le 4 décembre 2013]. Consultable en ligne à la page : http://www.bdso.gouv. qc.ca

17. Hux JE, Ivis F, Flintoft $V$, Bica A. Diabetes in Ontario : determination of prevalence and incidence using a validated administrative data algorithm. Diabetes Care. 2002;25(3): 512-516.

18. Jean S, Candas B, Belzile E et collab. Algorithms can be used to identify fragility fracture cases in physician-claims databases. Osteoporos Int. 2012;23(2):483-501.

19. Tu K, Mitiku T, Lee DS, Guo H, Tu JV. Validation of physician billing and hospitalization data to identify patients with ischemic heart disease using data from the Electronic Medical Record Administrative data Linked Database (EMRALD). Can J Cardiol. 2010;26(7):e225-e228.

20. Quan H, Khan N, Hemmelgarn BR et collab. Validation of a case definition to define hypertension using administrative data. Hypertension. 2009;54(6):1423-1428.

21. Tu K, Campbell NRC, Chen ZL, Cauch-Dudek KJ, McAlister FA. Accuracy of administrative databases in identifying patients with hypertension. Open Medicine. 2007;1(1):e18-e26.

22. Lee LM, Teutsch SM, Thacker SB, St. Louis ME. Principles and Practice of Public Health Surveillance. New York (NY) : Oxford University Press; 2010.

23. Blais C, Hamel D, Rinfret S. Impact of socioeconomic deprivation and area of residence on access to coronary revascularization and mortality after a first acute myocardial infarction in Quebec. Can J Cardiol. 2012;28(2):169-177.

24. Aube-Maurice J, Rochette L, Blais C. Associations divergentes entre l'incidence de l'hypertension artérielle et la défavorisation selon le mode d'identification des cas. Maladies chroniques et blessures au Canada. 2012;32(3):136-146.

25. Jean S, Bessette L, Belzile EL et collab. Direct medical resource utilization associated with osteoporosis-related nonvertebral fractures in postmenopausal women. J Bone Miner Res. 2013;28(2):360-371.
26. Robitaille C, Dai S, Waters C et collab. Diagnosed hypertension in Canada : incidence, prevalence and associated mortality. CMAJ. 2012;184(1):e49-e56.

27. Robitaille C, Bancej C, Dai S et collab. Surveillance of ischemic heart disease should include physician billing claims : populationbased evidence from administrative health data across seven Canadian provinces. BMC Cardiovasc Disord. 2013;13(1):88.

28. German RR, Lee LM, Horan JM, Milstein RL, Pertowski CA, Waller MN. Updated guidelines for evaluating public health surveillance systems: Recommendations from the Guidelines Working Group. MMWR Recomm Rep. 2001;50(RR-13):1-35.

29. Tamblyn R, Lavoie G, Petrella L, Monette J. The use of prescription claims databases in pharmacoepidemiological research: the accuracy and comprehensiveness of the prescription claims database in Quebec. J Clin Epidemiol. 1995;48(8):999-1009.

30. Institute of Medicine. A nationwide framework for surveillance of cardiovascular and chronic lung diseases. Washington (DC) : The National Academies Press; 2011.

31. St Sauver JL, Grossardt BR, Yawn BP et al. Data resource profile : the Rochester Epidemiology Project (REP) medical records-linkage system. Int $\mathrm{J}$ Epidemiol. 2012;41(6):1614-1624.

32. Centers for Disease Control and Prevention. NCHS Data Linkage Activities [Internet]. Centers for Disease Control and Prevention; 2014 [consultation le 31 mars 2014]. Consultable en ligne à la page: http://www. cdc.gov/nchs/data_access/data_linkage_ activities.htm

33. Australian Institute of Health and Welfare. Key indicators of progress for chronic disease and associated determinants: data report. Canberra (AUS) : Australian Institute of Health and Welfare; 2011.

34. Department of Health. Public Health Surveillance: Towards a Public Health Surveillance Strategy for England. London (UK) : Department of Health; 2012.
35. Paul C. Creating and using CCHS files to link survey responses to health service utilization data - Ontario's experience. Communication présentée à Explaining the miracle! Statistics and analysis in public health. $15^{\mathrm{e}}$ congrès de l'Association of Public Health Epidemiologists in Ontario (APEO); 14-16 oct. 2007; Ottawa (Ont.).

36. Lix L, Yogendran M, Burchill $C$ et collab. Defining and validating chronic diseases: an administrative data approach. Winnipeg (Man.): Manitoba Centre for Health Policy; 2006.

37. Lambert L, Blais C, Hamel D et collab. Evaluation of care and surveillance of cardiovascular disease: can we trust medico-administrative hospital data?. Can J Cardiol. 2012;28(2):162-168.

38. de Torbal A, Boersma E, Kors JA et collab. Incidence of recognized and unrecognized myocardial infarction in men and women aged 55 and older: the Rotterdam Study. Eur Heart J. 2006;27(6):729-736.

39. Ministère de la Santé et des Services sociaux du Québec (MSSS). Regards sur le système de santé et de services sociaux du Québec. Québec (Qc) : MSSS; 2009.

40. Institut canadien d'information sur la santé (ICIS). Base des données nationales sur les médecins 2010-2011 - publication des données. Ottawa (Ont.) : ICIS; 2013.

41. Pineault R, Provost S, Hamel M, Couture A, Levesque JF. L'influence des modèles d'organisation de soins de santé primaires sur l'expérience de soins de patients atteints de différentes maladies chroniques. Maladies chroniques et blessures au Canada. 2011;31(3):120-132.

42. Choi BCK. La surveillance épidémiologique au $21^{\mathrm{e}}$ siècle sous diverses optiques. Maladies chroniques au Canada. 1998;19(4):159-166.

43. Blais C, Rochette L, Hamel D, Poirier P. Prévalence, incidence, conscience et contrôle de l'hypertension dans la province de Québec : perspective des données administratives et des données d'enquête. Revue canadienne de santé publique. 2014;105(1):e79-e85.

44. Ouhoummane N. Impact du diabète sur la mortalité à la suite d'une hospitalisation pour un premier infarctus aigu du myocarde au Québec [thèse]. Québec (Qc) : Université Laval; 2010. 